\title{
Smart city information processing under internet of things and cloud computing
}

\author{
Peng Su${ }^{1} \cdot$ Yuanyuan $\mathrm{Chen}^{2} \cdot$ Mengmeng $\mathrm{Lu}^{1}$
}

Accepted: 22 June 2021 / Published online: 3 August 2021

(c) The Author(s), under exclusive licence to Springer Science+Business Media, LLC, part of Springer Nature 2021

\begin{abstract}
This study is to explore the smart city information (SCI) processing technology based on the Internet of Things (IoT) and cloud computing, promoting the construction of smart cities in the direction of effective sharing and interconnection. In this study, a SCI system is constructed based on the information islands in the smart construction of various fields in smart cities. The smart environment monitoring, smart transportation, and smart epidemic prevention at the application layer of the SCI system are designed separately. A multi-objective optimization algorithm for cloud computing virtual machine resource allocation method (CC-VMRA method) is proposed, and the application of the IoT and cloud computing technology in the smart city information system is further analysed and simulated for the performance verification. The results show that the multi-objective optimization algorithm in the CC-VMRA method can greatly reduce the number of physical servers in the SCI system (less than 20), and the variance is not higher than 0.0024 , which can enable the server cluster to achieve better load balancing effects. In addition, the packet loss rate of the Zigbee protocol used by the IoT gateway in the SCI system is far below the $0.1 \%$ indicator, and the delay is less than $10 \mathrm{~ms}$. Therefore, the SCI system constructed by this study shows low latency and high utilization rate, which can provide experimental reference for the later construction of smart city.
\end{abstract}

Keywords Smart city $\cdot$ Internet of Things $\cdot$ Cloud computing $\cdot$ Smart city information system $\cdot$ Smart epidemic prevention

Peng Su

596734835@qq.com

1 Scientific Research Planning Division, Shandong Labor Vocational and Technical College, Jinan 250022, Shandong, China

2 College of Electronic Information Engineering, Rizhao Polytechnic, Rizhao 276826, Shandong, China 


\section{Introduction}

With the rapid development of science and technology, people's living standards have also improved significantly. Since the term "smart city" was proposed in 2009 , its development has attracted much attention. Driven by economic globalization, the process of urbanization in various countries has accelerated significantly, and the urbanization rate has increased year by year. However, the excessively accelerated urbanization process has also brought great pressure on global resources and the environment. Among them, resource shortages, environmental pollution, traffic congestion, and other "urban diseases" have become increasingly prominent, and have become the primary problems that plague urban construction $[1,2]$. As the rapid development of urbanization is subject to the influence of factors such as new technologies, innovation capabilities, systems and management levels, the healthy and sustainable development of cities is restricted by the above-mentioned multiple factors. Therefore, the development and improvement of information technology of smart city has become the focus of attentions of many scholars in related fields.

As one of the manifestations of new urbanization, smart city is the inevitable result of the development of information technology to a certain extent. The construction of smart city is also an optimization and upgrading of the industrial structure of the city. Through the comprehensive and transparent perception of information, the wide and safe transmission of information, and the smart and efficient processing of information, it improves the efficiency of city management and operation, elevates the level of city services, and promotes the city. The sustainable and leap-forward development of the city will finally build a new urban development form, which makes the city realize automatic perception and effective decision-making and regulation, and allows citizens to feel the smart services and applications brought by smart city [3]. Information technology is the infrastructure of smart city, and IoT and cloud computing are undoubtedly the two core technical hotspots in its construction. IoT technology is based on radio frequency identification (RFID) and other sensing devices. The IoT technology can establish the connection between the sensing device and the Internet, and realize information communication and exchange, and build a smart management environment for object identification and tracking through the IoT gateway [4-6]. The main points of IoT are effective perception, extensive interconnection, in-depth smart analysis and processing, and personalized experience. As a new Internetbased software and hardware service model, the cloud computing aims to provide users with fast, dynamic, and easy-to-expandable virtualized resource services through minimal management costs and configurable computing resources, such as servers, information centres, networks, and applications [7]. Only a simple terminal device is required to pass identity verification and then apply software and hardware services, such as desktop systems and open platforms. The software, hardware, and data are all in the cloud computing centre. With the development of IoT and cloud computing technology, its application areas have become more and more extensive. For the development and construction of smart city, it has 
a more intuitive display in the fields of road transportation, urban infrastructure construction, medical services, and logistics services [8]. The concept of smart city construction with new-generation technologies (such as IoT and cloud computing) as the core has become a brand new model for future urban development [9]. As an inevitable product of the development of urbanization, the construction of smart city is also feasible from the perspective of technology and management, which is conducive to improving the level of urban information management and promoting the development of national high-end industries.

To sum up, the construction of smart cities is inseparable from the support of the IoT and cloud computing technology, but the situation of information islands is becoming more and more serious with the development of intelligence in various fields, so that the construction of smart cities is developing in the direction of effective sharing and interconnection, which shows extremely important meaning. The innovations of this research can be summarized as follows. Firstly, in response to the current new crown epidemic, the physical networks and cloud computing technology are applied in the construction of medical infrastructure of smart city, which shows high performance of information security and confidentiality. Secondly, the application layer of the SCI system is specifically designed from the perspectives of smart transportation, smart environment monitoring, and smart epidemic prevention. Thirdly, a CC-VMRA method based on multi-objective optimization algorithms is proposed to solve the data and information sharing in the construction of smart city information systems. It shows clearer reference value for the later establishment of smart city information system.

\section{Recent related work}

\subsection{The status of IoT in the construction of smart city}

The IoT perception layer that the SCI system relies on provides multiple sources of information with a variety of types and a large volume. It is necessary to build a unified data access and collection mechanism, so as to provide heterogeneous, multidimensional, massive, multi-temporal, and multi-observation model information for the social and economic life of people, which has been researched by many scholars. Bresciani et al. (2018) modelled the data of multiple foreign IoT smart city project alliances to realizing close correlation between the development of the company and the rapid development of urbanization; the author emphasized that the knowledge management ability indirectly improves the flexibility of the alliance through the information and communication technology capabilities of the enterprise, and recommended that the managers of multinational companies design knowledge management tools and develop new information and communication technology skills [10]. Qian et al. (2019) used IoT technology to promote the construction of urban infrastructure, which makes the urban economy grow sustainably and people's living standards improve significantly [11]. Chen et al. (2020) used the convolutional neural network (CNN) in deep learning to consider the evacuation function in the design process of smart city public construction; it was found that the evacuation process took the shortest time after the 
model training, which can have a good effect on the construction of smart cities [12]. Watson et al. (2020) discussed and estimated the big data-driven decision-making process in the knowledge-based city economy by comprehensively analysing the existing achievements and basis of IoT smart city; and it was found that although IoT has made a great contribution to the construction of smart city, it still can't meet the needs of smart city development [13].

\subsection{The status of cloud computing in the construction of smart city}

Cloud computing is mainly manifested in the construction of smart city as an urban data centre, which is the core of the SCI system and has been researched by many scholars. Lv et al. (2018) proposed a new government service platform using threedimensional geographic information system (GIS) and cloud computing technology to enable the three-dimensional analysis and visualization of city information on the smart city platform; and it was found that the system can perform more accurate monitoring and evaluation of urban disasters and environmental protection, intelligent transportation, and urban resource centres in smart cities [14]. Hossain et al. (2018) found that although the traditional cloud computing method can provide the largest computing and storage facilities to support data processing, its latency is relatively high, so the edge computing was applied in the construction of smart city; finally, it was found that processing raw IoT data on edge devices are effective in terms of latency, providing context awareness for smart city decision makers in a seamless manner [15]. Giannakoulias et al. (2019) studied the data security issues faced in cloud computing environments and introduced some of the most important security threats in cloud computing, as well as key recommendations on how to deal with these threats, including security standards and certifications, service provider audits, security application programming interfaces (APIs), transport layer protection, identity verification, encryption key management, and cloud service agreements [16]. Javadzadeh et al. (2020) believed that the technology used to realize smart city is usually based on cloud computing, but such technology is accompanied by unreliable latency, lack of mobility support and location awareness; and the fog computing was applied to the smart city to explore the research trend and development direction of smart city [17].

In summary, the IoT and cloud computing are the key technical support in the construction of smart cities. There are many studies on the application of these two core technologies to the construction of smart cities. Although the IoT and cloud computing are widely used in the construction of smart cities, their specific applications in smart cities are not clear, and they lack a mature theoretical system and complete index requirements. Therefore, the specific application layer, network layer, and perception layer of the SCI system are analysed in this study, which is of great significance to the construction of smart cities. 


\section{Methods}

\subsection{Analysis on construction of $\mathrm{SCl}$ system}

Smart city refers to the use of new-generation of information technologies such as IoT and cloud computing to fully perceive and effectively integrate and process various data and information within the physical city, to make objects smart and to coordinate various systems in the city through the smart management of the government, providing a good development space for enterprises in different fields in the city and a more convenient and high-quality living environment for urban residents, so that the urban economy, society, and environment can achieve sustainable development $[18,19]$. The characteristics of SCI system are shown in Fig. 1.

As a large and complex project, smart city needs to have a strategic foresight and conform to the personality of each city when a master plan is formulated. At the same time, the relationship among various fields should be fully considered, and the plan for important areas should be formulated to guide the actual construction of the smart city. Although the resources and development status of each city are different, the SCI system can be divided into three categories from the perspective of networking and informationization: urban environment, social management and services, and economic development based on the objectives.

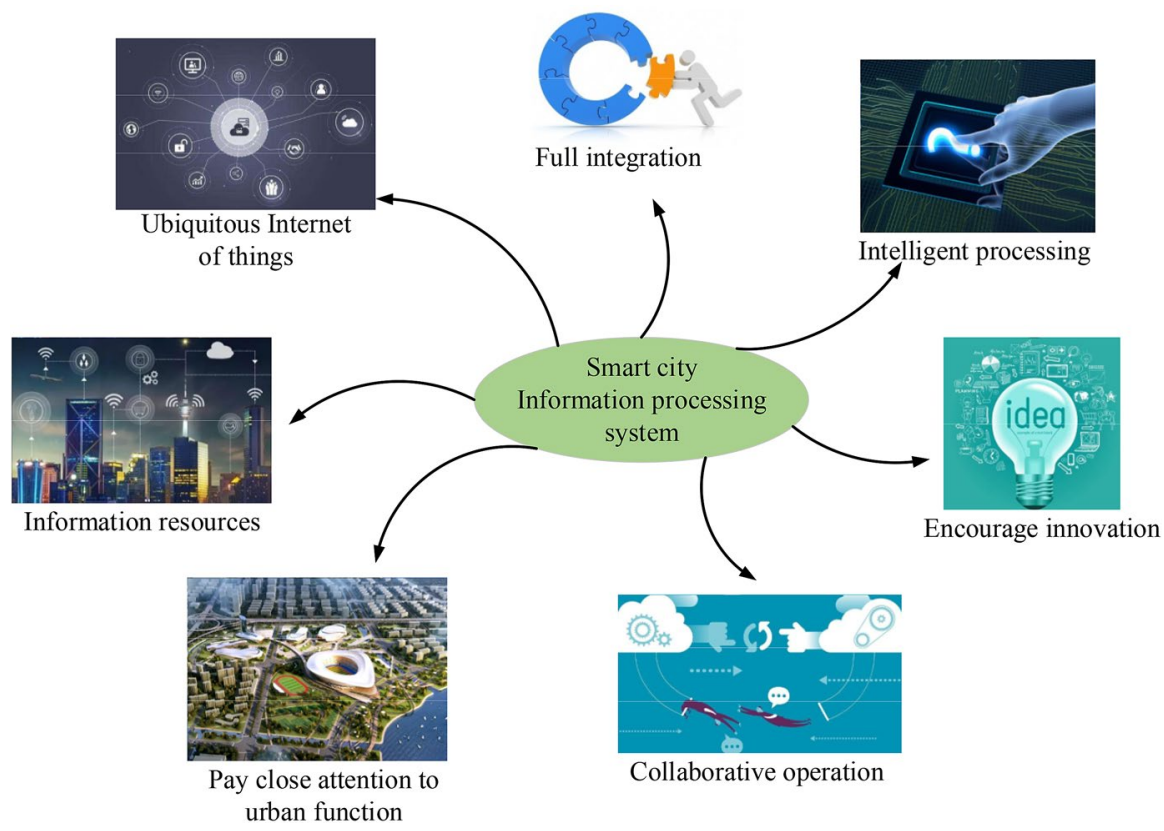

Fig. 1 The characteristics of SCI system 


\subsection{Construction and study on $\mathrm{SCl}$ system}

The construction of the SCI system is based on meeting the actual informatization needs of the city to establish a perceptual, smart, integrated, and open smart city informatization system, which can provide a "plug-and-use" smart integrated platform, achieving rapid allocation with high flexibility and scalability, and guaranteeing the long-term stable operation of the system through some security measures [20]. From a functional perspective, the SCI system is divided into environmental smart monitoring, smart transportation, smart security, smart fusion processing, public information publishing services, and geographic information; from the perspective of system architecture, the SCI system includes application system, network transmission system, and collection terminal system (as shown in Fig. 2).

In the SCI system, the terminal data information collection layer is the basis for the formation of smart city and the construction of the information system. The application layer is an important link for the full use of system functions, and the network layer is the connection of the SCI system. In the construction of the SCI system, the smart environment monitoring, smart transportation, and smart epidemic prevention of the application layer are separately described.

\subsubsection{Smart environment monitoring}

In the environmental monitoring of the smart city, the environmental monitoring units and modules (such as smoke and harmful gases) set up in the park enable monitoring of the real-time environmental information (temperature, humidity, and harmful gas content) of each district in the city. Such data are collected to the application support platform through the transmission network. The smart environmental monitoring system mainly includes two parts: environmental data collection and environmental-related data receiving and processing. Of which, the environmental data collection mainly contains two sub-parts: sensors and wireless transmission. The environmental-related data receiving and processing includes wireless data receiving and processing. The sensors arranged at each node in the city collect the environmental data and send it to the environmental data processing part by the transmission system. After data processing and analysis in the environmental data processing part, relevant operations are automatically triggered if the environmental data exceeds the safe range, and the transmission system sends the data to the environmental data processing part. Related management personnel and the system send alarm information and call the corresponding camera for video viewing. The schematic diagram of smart environmental monitoring is shown in Fig. 3.

In smart environmental monitoring, the collection of environmental data is realized by deploying multiple monitoring nodes, and the collected data are sent to the relay node through wireless Zigbee technology [21] and then collected into the processing system through a wired or wireless network, realizing the analysis, processing, and storage of data. In conjunction with the video network, the analysis results are sent to the relevant personnel of the park management, so that the monitoring efficiency and the response processing speed of the personnel are significantly improved. 


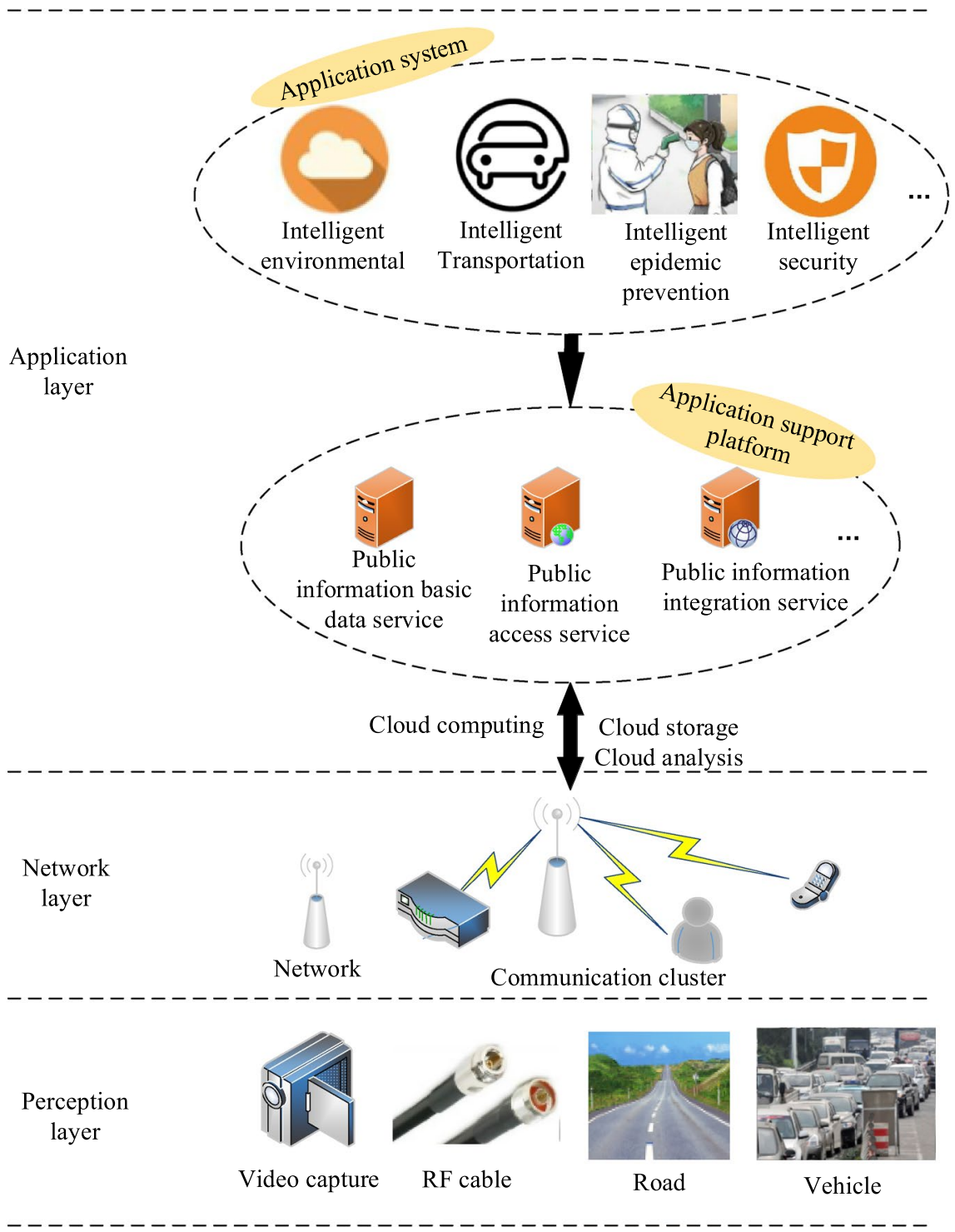

Fig. 2 Structure of SCI system

\subsubsection{Smart transportation}

In the design and application of smart transportation of the SCI system, the global position system (GPS) module and RFID module integrated on the vehicles in each district of the city are adopted to monitor the location of the vehicles in each district in the city in real time. In addition, the real-time situation of each road section in the city is counted and summarized to the application support platform through 


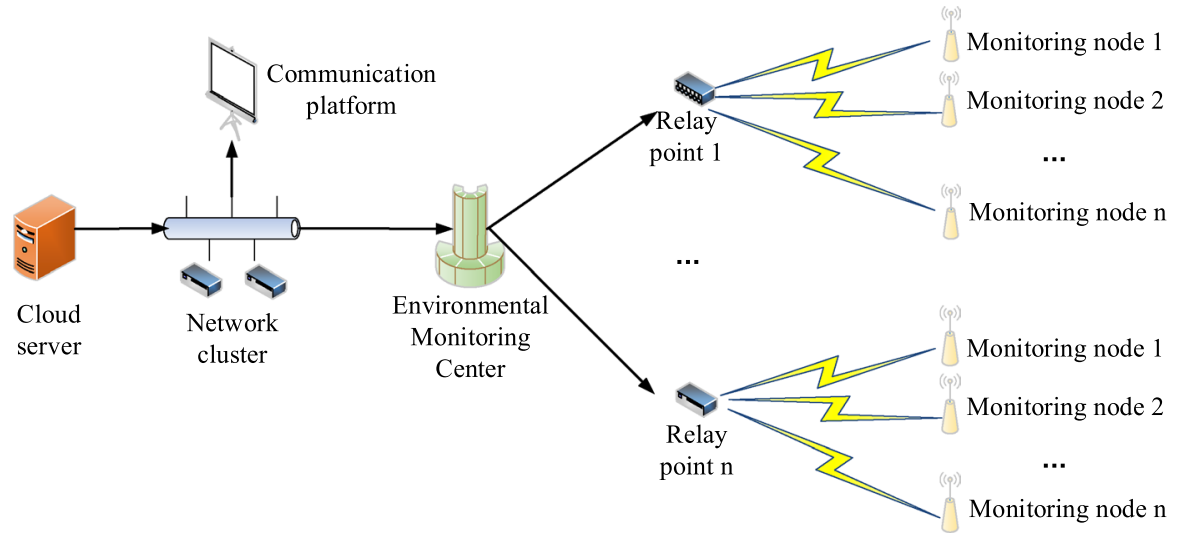

Fig. 3 The schematic diagram of smart environmental monitoring in the SCI system

the transmission network. After authentication, control canter personnel and users can call up the real-time information of various road sections, and then select the most suitable route or venue for vehicle-related operations. Vehicles certified in the city can pass RFID certification to finally achieve free parking in and out of various areas of the city. The schematic diagram is shown in Fig. 4.

In the SCI system, the GPS module integrated on the smart transportation vehicles can realize real-time monitoring of the vehicles in the park. When traffic congestion occurs on some roads in the city, digital clusters and integrated communications platform can send the congestion information to the relevant personnel of smart traffic management in a timely manner, so that the congestion in the city can be effectively dealt with.

\subsubsection{Smart epidemic prevention}

With the global spread of COVID-19, the establishment of a smart epidemic prevention system in the SCI system is of great significance to the protection of human health. In this study, the smart epidemic prevention refers to the use of the communication network layer to track the trajectories of people and real-time monitoring of human body temperature and other conditions, to take advantage of the large amount of data on the cloud storage platform and the wide range of cloud analysis, and to utilize the video resources comprehensively, achieving the effect of monitoring the deployment of the epidemic. In addition, video monitors are installed in key areas of the smart city to monitor the conditions of various road sections, communities, and buildings in the city in real time. The distribution of all video resources can be displayed on the GPS map visibly, based on which the operator can select the specific area by marking a circle with mouse to quickly open the video image, realizing the video deployment control based on the GPS map. Based on a large number of video behaviour analysis, the utilization and reliability of the system are improved. The smart epidemic prevention monitoring system is shown in Fig. 5. 


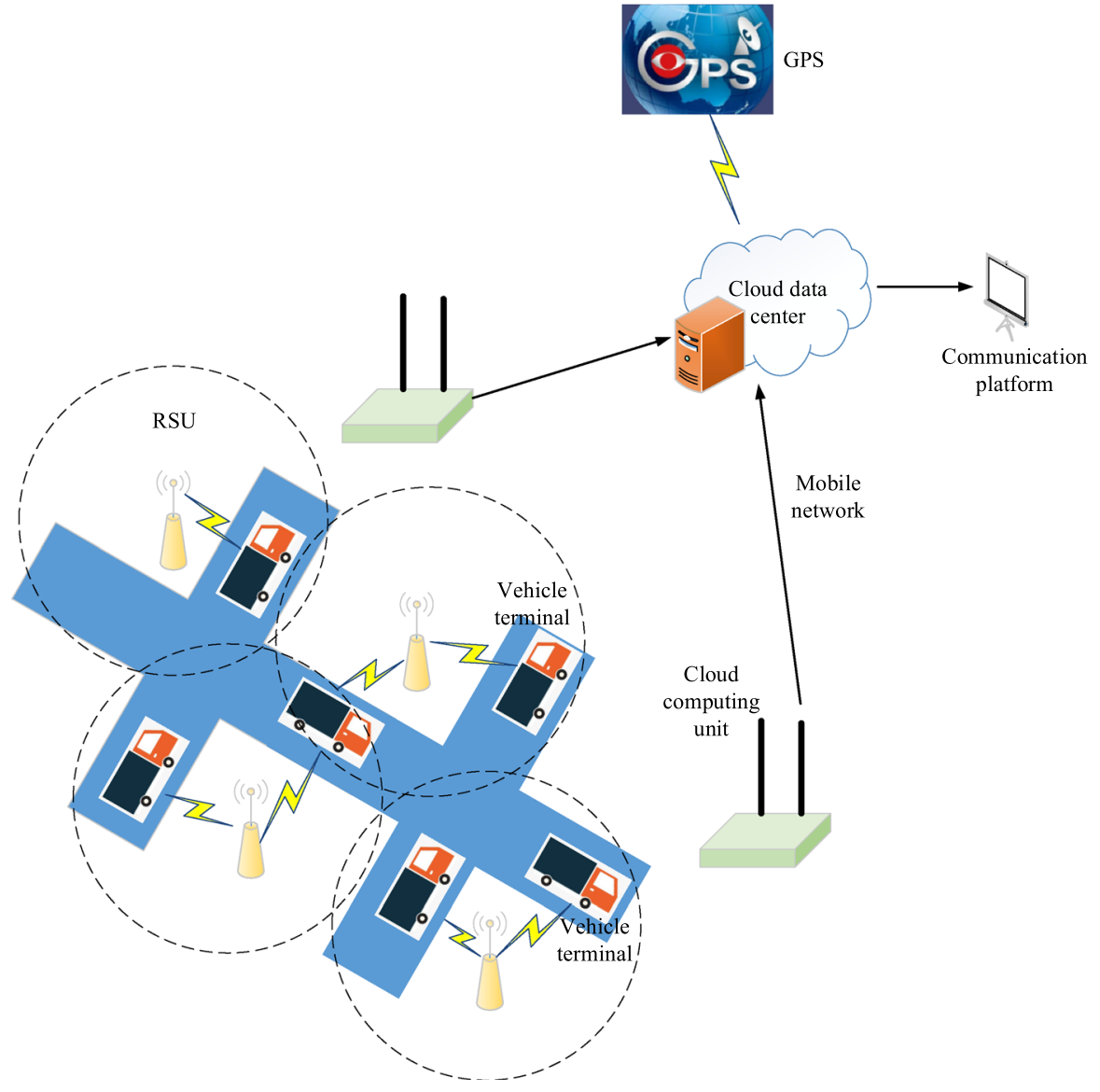

Fig. 4 The schematic diagram of smart transportation in SCI system

In the design of smart environment monitoring, smart transportation, and smart epidemic prevention of the application layer for the SCI system, the supporting platform of each sub-application adopts cloud computing, cloud storage, and cloud analysis to provide public basic data information services, public information wireless, wired access services, public integrated information services, and public information exchange services for various fields in smart city. In this way, the high-definition monitoring, predictive warning, smart analysis, and smart control of various fields in the city can be realized.

\subsection{Critical technologies used for $\mathrm{SCl}$ system}

In this study, artificial intelligence technologies such as cloud computing and IoT are mainly used in the construction of the SCI system. The urban data centre is the core of the SCI system. Building an urban cloud data centre based on the cloud 


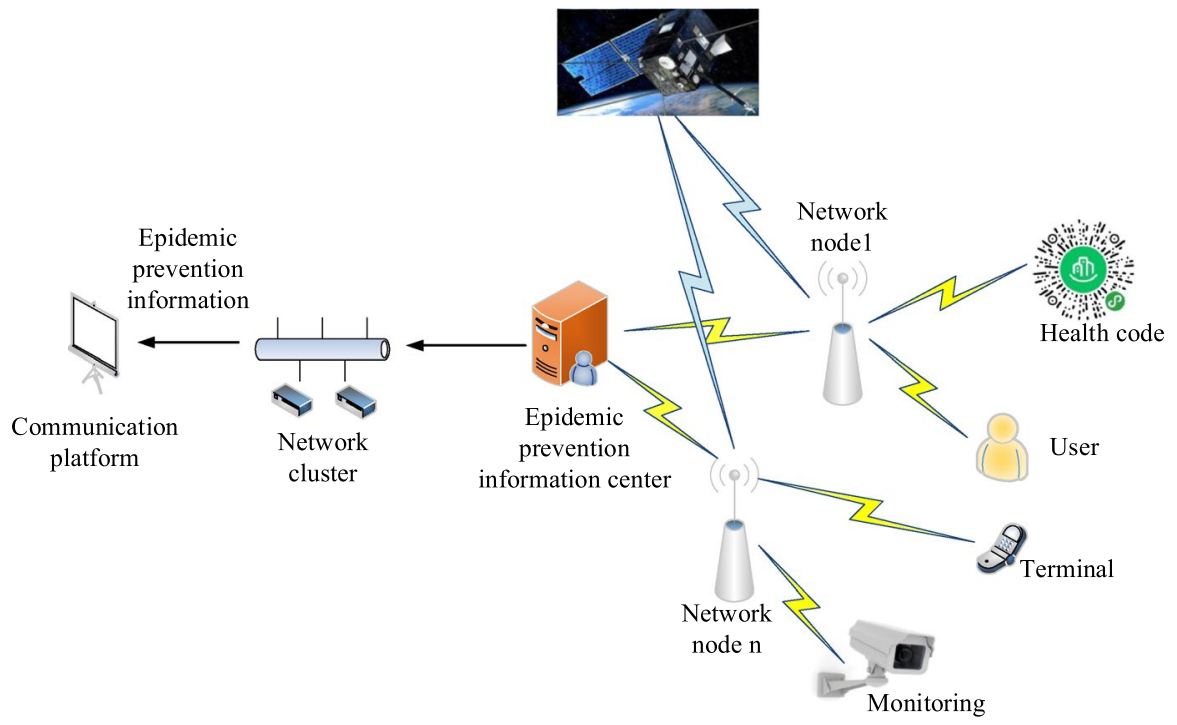

Fig. 5 The schematic diagram of smart epidemic prevention monitoring system of SCI system

technology can not only improve the availability of the SCI system, but also maximize the utilization of data centre hardware resources. However, how to apply the cloud computing and IoT technology to effectively manage and efficiently dispatch the urban resources and to ensure the balanced load performance and low energy consumption of cloud data centres has become a difficult point in the current research on the construction of smart cities.

\subsubsection{Cloud computing technology used in $\mathrm{SCl}$ system}

In the construction of smart city, it is difficult to implement unified physical resource management due to the differences and poor compatibility between the hardware equipment of the urban data centre. The cloud data centre uses virtualization technology to uniformly construct the computing resources, storage resources, and network resources into a virtual resource pool. The virtual resource management technology is adopted to realize automatic allocation and scheduling, dynamic expansion, and automatic deployment of cloud computing resources on demand, and users can obtain the resources on demand, ultimately providing a scientific and feasible plan for the unified management of resources [22]. The virtualized structure of urban resources is shown in Fig. 6.

In the construction of smart city, CC-VMRA is a multi-objective vector issue, that is, a multi-objective combinatorial optimization problem. In the multi-target virtual machine allocation, the available resource of each physical node is a d-dimensional vector, and each dimension represents a certain resource, such as central processing unit (CPU), memory, and network bandwidth. The resource of each virtual machine is also a d-dimensional vector. The goal is to put multiple 


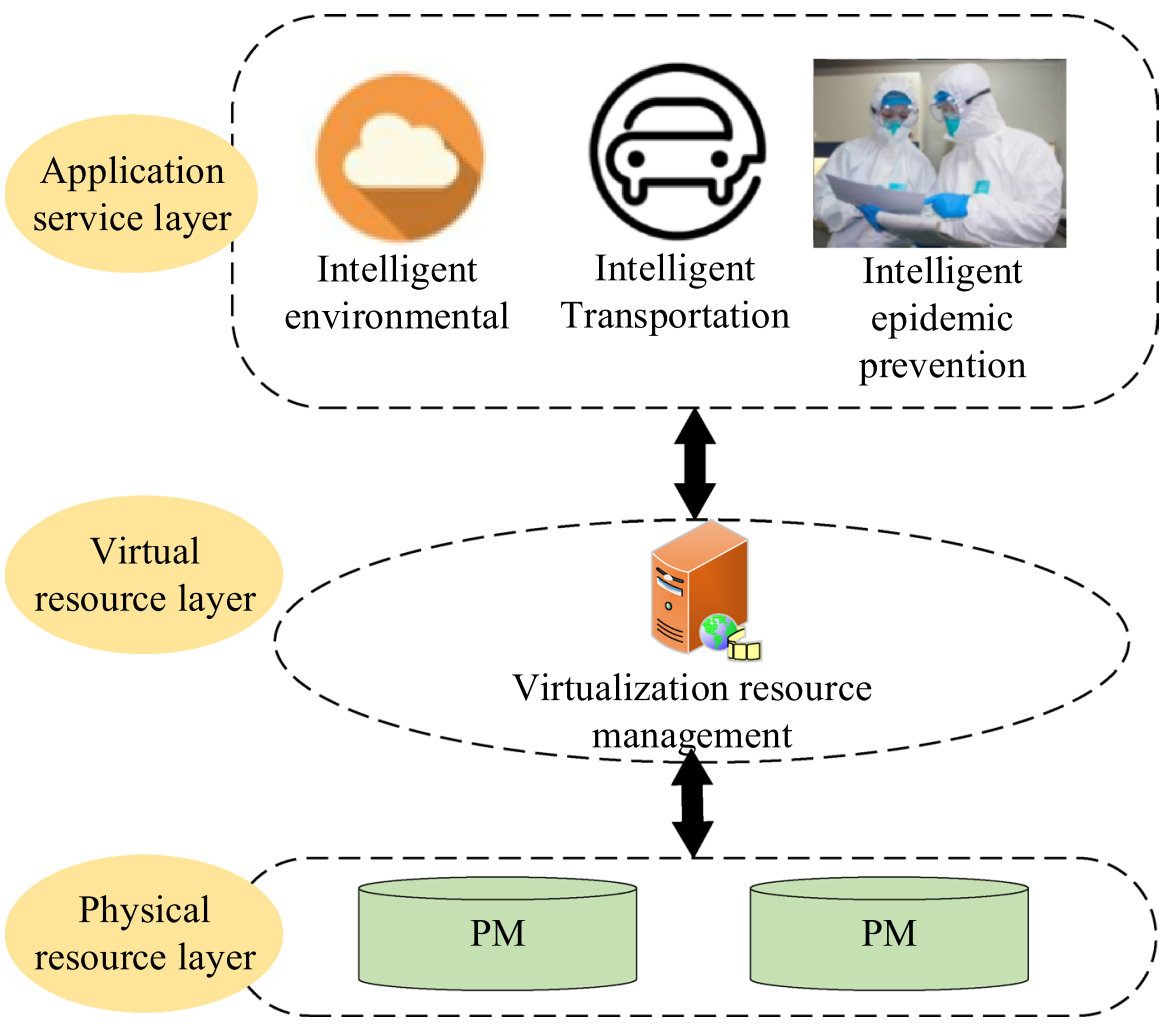

Fig. 6 Schematic diagram of virtualized structure of urban resources in SCI system

virtual machines into multiple physical nodes, and finally achieving the goal of minimizing the number of physical nodes and minimizing load variance. The description of CC-VMRA of multi-objective optimization algorithm is as follows:

$$
\begin{array}{r}
f_{\mathrm{PN}}=\min \sum_{f} C_{f} \\
f_{\mathrm{LB}}=\min \frac{\sum_{d} D \xi_{i}}{d}
\end{array}
$$

In Eq. (1), $f_{\mathrm{PN}}$ refers to the number of occupied physical nodes, and $f_{\mathrm{LB}}$ refers to the balanced load variance of the server cluster. The value of $C_{f}$ is given as follows:

$$
C_{f}=\left\{\begin{array}{cc}
1, & \text { Physical node } \ddot{1} \text { use } \\
0 & \text { else }
\end{array}\right.
$$

In Eq. (2), $D \xi_{i}$ refers to the $i$-dimensional variance, and $d$ refers to the total dimensionality. The expression is $D \xi_{i}$ as follows: 


$$
D \xi_{i}=\frac{\sum_{n}\left(p_{i j}-\bar{p}_{i}\right)^{2}}{n}
$$

In the above equation, $n$ refers to the number of physical nodes, $\bar{p}_{i}$ refers to the average value of the $i$-th dimension performance characteristics of all physical nodes. The performance characteristics are normalized values, which are equal to the remaining allocation of the $i$-th dimension resources in the physical nodes divided by the total $i$-dimensional resources. $p_{\mathrm{ij}}$ refers to the $i$-th dimension performance characteristics of physical node $j$.

$$
\begin{aligned}
& \sum_{i} V_{i}^{\mathrm{cpu}} * x_{i \rightarrow j} \leq P_{i}^{\mathrm{cpu}} \\
& \sum_{i} V_{i}^{\mathrm{mem}} * x_{j} \leq P_{j}^{\mathrm{mem}} \\
& \sum_{i} V_{i}^{I / O} * x_{j} \leq P_{j}^{I / O}
\end{aligned}
$$

In Eqs. (5), (6), and (7), $x_{i \rightarrow j}$ refers to 1 when virtual machine $i$ is allocated to physical node $j$, otherwise it is $0 . V_{i}^{\mathrm{cpu}}, V_{i}^{\mathrm{mem}}$, and $V_{i}^{I / O}$ refer to the CPU, memory, and network bandwidth resources of virtual machine $i$, respectively; $P_{i}^{\mathrm{cpu}}, P_{j}^{\mathrm{mem}}$, and $P_{j}^{I / O}$ refer to the CPU, memory, and network bandwidth resources of physical node $j$, respectively. The application system $P$ may be deployed on $\mathrm{cm}$ virtual machines (VMs), and the performance indicators provided by the $\mathrm{cm}$ VMs to each application system should meet the service level agreement (SLA) [23] of each application system $P$, then below equation can be obtained:

$$
\sum_{\mathrm{cm}} V_{\mathrm{ip}} \geq S L A_{p}
$$

Equation (8) is used in the dynamic resource scheduling of various application fields in smart city. The performance is monitored by the resource monitor. When it is overloaded, new virtual machines are deployed to share the load. When the access drops, the VMs can be reduced under the precondition of satisfying Eq. (8). Based on the basic framework of virtual machine dynamic scheduling, a virtual machine dynamic scheduling algorithm based on application service performance is used to solve the virtual machine load or low utilization rate. Among them, the pseudo code of the virtual machine dynamic scheduling algorithm with low virtual resource load is shown in Fig. 7.

Note: in the pseudo code algorithm, get $\operatorname{VMUR}()$ and get $\mathrm{AS}()$ refer to the returns the utilization rate of the virtual machine and the returns the application service flag deployed on the virtual machine, respectively; PI represents the returns the available performance indicators of the virtual machine; get New VM type() represents returning to the type of new virtual machine to be added (computing resource consuming type, memory resource consuming type); Add New VM() refers to allocating 
Fig. 7 Pseudo code of virtual machine dynamic scheduling algorithm with low virtual resource load

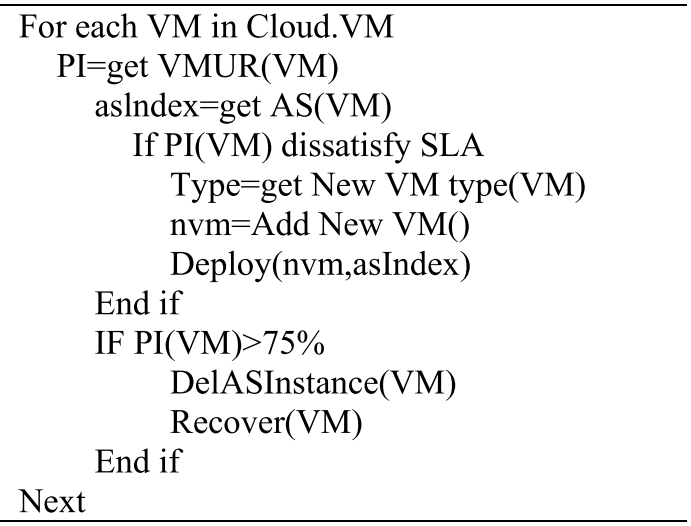

a new virtual machine; Deploy() means to deploy the belonging application service to the newly allocated virtual machine; DelASInstance() means to delete the application service instance on the virtual machine; and Recover() refers to recover the resources of virtual machine.

\subsection{2 loT technology in $\mathrm{SCl}$ system}

In the construction of smart city, IoT and ubiquitous networks provide heterogeneous, multi-dimensional, massive, multi-temporal, and multi-observation model information for people's social and economic life. Ubiquitous information comes from a wide variety of sensors, controllers, and computing terminals, and the technologies adopted by these devices lack compatibility and system planning. Then, how to realize the interconnection of various communication standards and the conversion of different data formats has become a problem that must be solved in the SCI system.

The IoT gateway is a device that bridges the perception network [24] and the Internet. Its main function is embodied in the access capability of the perception device and the ability to manage the perception device, and the conversion ability of the protocol is mainly reflected in the conversion from the own data transmission communication protocol of various devices such as Zigbee protocol to the transmission control protocol / internet protocol (TCPIP) [25]. In the functional analysis of the IoT gateway, the software architecture of the IoT gateway that supports multisensor information access and collaborative services can be divided into four levels from left to right: wide area access layer, protocol adaptation layer, and protocol conversion control layer, and perception access layer (Fig. 8).

In the wide area access network, the IoT gateway is required to have sufficient and reliable bandwidth to provide data services to the application layer; while in the protocol conversion and control layer, it mainly includes a remote management module, a control module, and a protocol conversion module. The function of the remote management module is to interface the management protocol with the management module in the platform, process the management request of the application layer, 


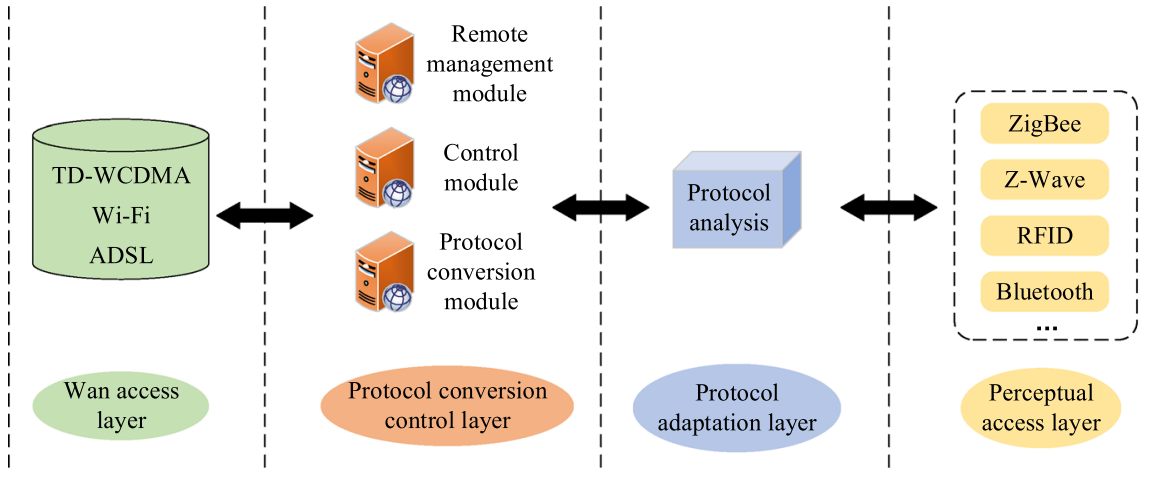

Fig. 8 Schematic diagram of IoT in SCI system

and generate the command after merging and forward it to the control module. The control module analyses the content of the command and sends it to the protocol adaptation layer through the protocol conversion module. The protocol conversion module also has high-level protocol conversion capabilities, which can convert the data collected by sensors into different application protocols. In the protocol adaptation layer, a unified format of data and signalling can be formed by analysing the communication protocols of different sensor nodes in the SCI system, or the control commands issued by the management platform can be customized to adapt to the data of different types of sensor nodes. The perceptual access layer is mainly equipped with rich sensor access capabilities, consisting of physical devices and driver service programs that cooperate with them.

\subsection{Performance test of $\mathrm{SCl}$ system}

The traditional priority matching heuristic (PMH) [26] and the single-objective clustering genetic algorithm (CGA) with the number of physical nodes [27] as the target are compared with the newly proposed CC-VMRA method based on the multi-objective optimization algorithm (the proposed algorithm), so as to verify the performance of the cloud computing virtual machine in the SCI system. The simulation is performed on the C1oudSim simulation program [28]. The specific parameters of each algorithm in the calculation and verification process are given as follows: the population size and evolution algebra are set to 30 and 1000, respectively, and the crossover rate and mutation rate are set to 0.6 and 0.02 , respectively. The simulation experiment parameters of VM allocation algorithm are shown in Table 1.

In the IoT application system of the SCI system, the performance of the IoT gateway is mainly reflected in PLR and latency [29]. The experimental network of this study includes 8 Zigbee sensor nodes, built-in temperature, humidity, and illuminance sensors. The Ad-Hoc mode networking [30] is adopted, and the IoT gateway is undertaken as the sink node directly. In this simulation experiment, the data set is obtained from the sensor nodes, then the simulation data set is obtained after 1000 iterations, and finally the data set is allocated according to the leave-one method for 
Table 1 The simulation experiment parameters of VM allocation algorithm

\begin{tabular}{llll}
\hline $\begin{array}{l}\text { Number of } \\
\text { physical nodes }\end{array}$ & The generation of evolution & $\begin{array}{l}\text { The number } \\
\text { of VMs }\end{array}$ & VM allocation algorithm \\
\hline 25 & 80 & 20 & PMH, CGA, and the proposed algorithm \\
25 & & 30 & \\
25 & 40 & \\
25 & 50 & \\
25 & 60 & \\
\hline
\end{tabular}

training and testing. The PLR and latency characteristics of the gateway are tested by comparing the Zigbee networking in this study with the Irregular Repetition Slotted Aloha (IRSA) protocol [31]. The specific simulation experiment configurations are mainly considered from two aspects: hardware and software. In the software, the operating system is Linux 64bit, the Python version is Python 3.6.1, and the development platform is PyCharm; in the hardware, the central processing unit (CPU) is Intel core i7-7700@4. 2 GHz 8 core, memory is Kingston ddr4 $2400 \mathrm{MHz} 16 \mathrm{G}$, and the graphics processing unit (GPU) is Nvidia GeForce $10608 \mathrm{G}$.

\section{Results and discussion}

\subsection{Results and analysis of CC-VMRA}

The proposed algorithm is compared with the PMH and CGA from the perspective of the number of physical nodes enabled and the variance of cluster load, and the results are shown in Figs. 9 and 10, respectively.

Figures 9 and 10 illustrate the results of the number of physical nodes enabled and the variance of the cluster load of each algorithm when the number of VMs are different. The number of physical nodes occupied by the VM deployment algorithm in this study is basically the same as the number of physical nodes occupied by the VM deployment algorithm based on CGA and is less than that of the PMH algorithm. The smaller the number of physical nodes, the more savings energy consumption. The overall load variance of the cluster of the VM deployment algorithm in this study is smaller than that of the other two VM deployment algorithms, and the variance is not higher than 0.0024 . The smaller the load variance, the smaller the server cluster load, and the better the equalization effect. Therefore, above results reveal that the VM deployment algorithm optimized by the multi-objective combination of this study can greatly reduce the number of physical servers in the SCI system and enable the server cluster to achieve better load balancing effects.

\subsection{Test performance analysis of IoT gateway}

In the specific test of the performance of the SCI system, the management platform configures the sensor node to send data at $1 \mathrm{~s}, 5 \mathrm{~s}, 10 \mathrm{~s}, 15 \mathrm{~s}$, and $20 \mathrm{~s}$ for 


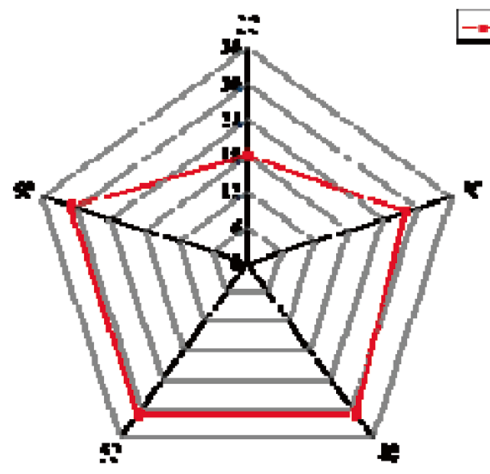

A

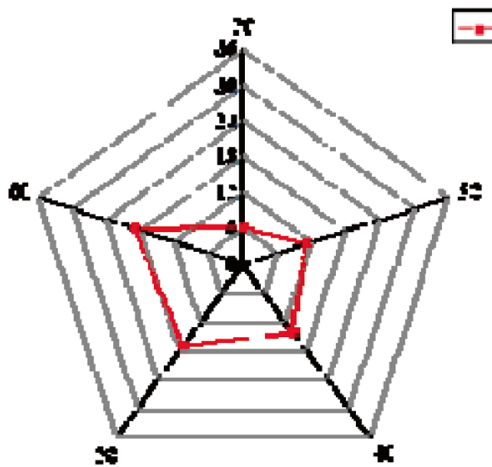

B

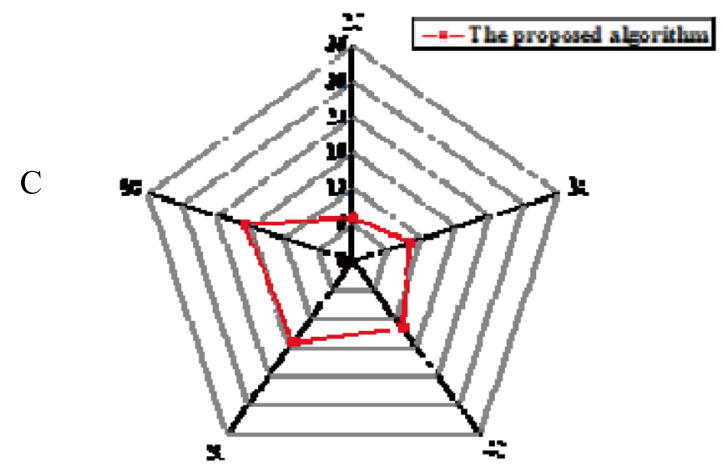

Fig. 9 The analysis results of the number of physical node enabled of various algorithms at different numbers of VMs (Figures A, B, and C showed the results of PMH, CGA, and the proposed algorithm, respectively)

$1 \mathrm{~h}$ to count the PLR, so as to determine whether PLR is calculated according to the serial number of the collected data packets. The final statistical result is shown in Fig. 11. It discloses that when the transmission period of the Zigbee protocol and the IRSA protocol is $1 \mathrm{~s}$, the PLR is $0.06 \% \sim 0.07 \%$. With the increase of the transmission interval, the PLR of the two protocols decreases, but it is obvious that the Zigbee protocol used in this study has a lower PLR, which is far below the maximum PLR $0.1 \%$ index specified by the CCSA standard.

The latency generated by the IoT gateway is analysed further to determine whether the received data exceeds the timeliness of the real-time system. As shown in Fig. 12, both protocols have reached the requirement that the latency is less than $10 \mathrm{~ms}$ with the increase of time. However, it is obvious that the Zigbee protocol in this study can achieve the latency of less than $10 \mathrm{~ms}$ in a shorter time. Therefore, the Zigbee protocol used by the IoT gateway in the SCI system of this study meets the requirements on both latency and PLR. 


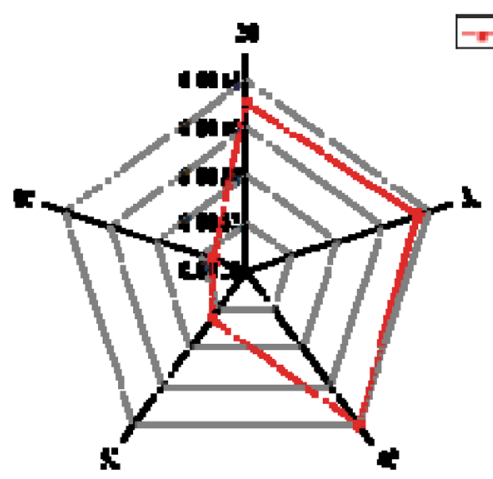

A

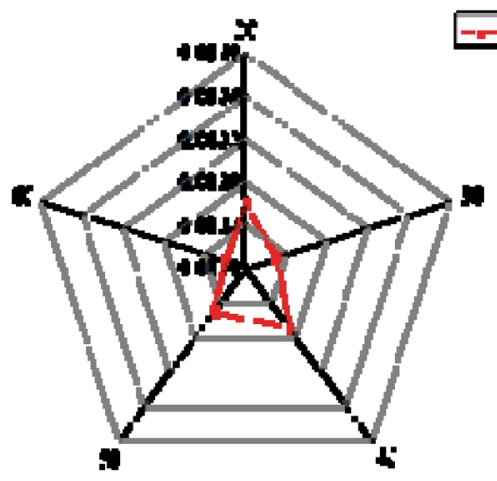

B

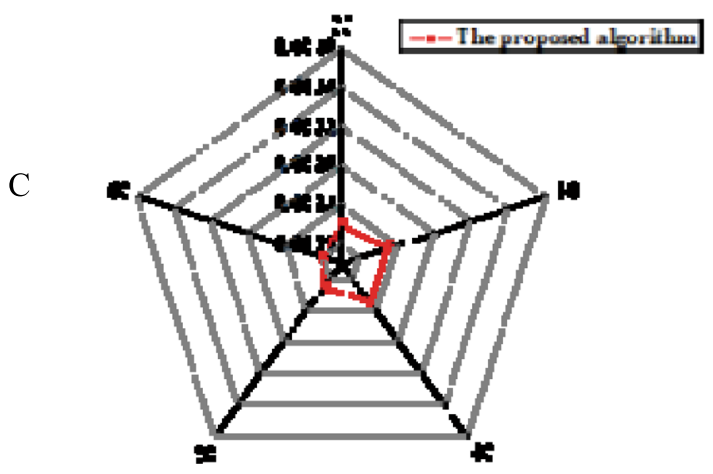

Fig. 10 The analysis results of the variance of cluster load of various algorithms at different numbers of VMs (Figures A, B, and C showed the results of PMH, CGA, and the proposed algorithm, respectively)

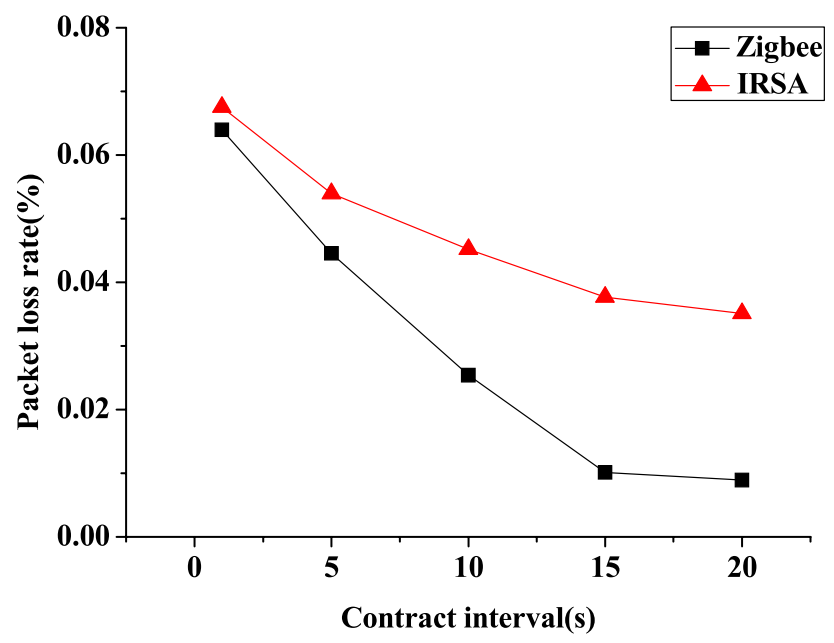

Fig. 11 Analysis results of the relationship between IoT gateway packet sending interval and PLR under different protocols 


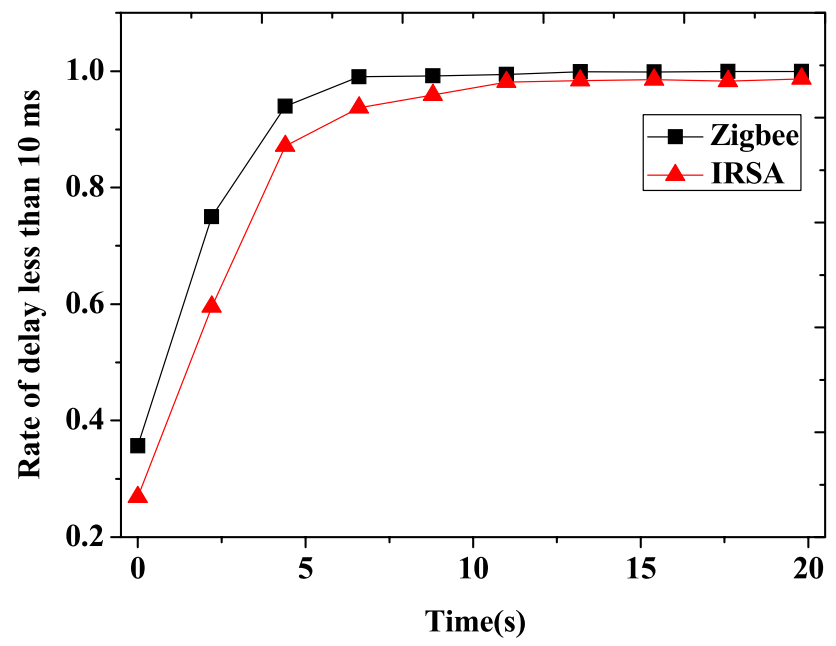

Fig. 12 The latency analysis results of IoT gateways under different protocols

\subsection{Discussion}

After the information processing of smart cities is analysed, a CC-RMRA method is proposed based on a multi-objective optimization algorithm. After the algorithm proposed is compared with PMH [26] and CGA [27], it is found that the number of physical nodes in the proposed algorithm is small and the variance is not higher than 0.0024 . This is because the algorithm uses the same number of physical nodes and realizes higher utilization rate of server resources, with higher accuracy and smaller variances. In addition, the effective utilization of server resources is increased to a greater extent, the number of active physical servers is reduced, and more energysaving effects are achieved. Therefore, the proposed CC-RMRA algorithm for multiobjective combination optimization can greatly reduce the number of physical servers in the smart city information system and enable the server cluster to achieve a better load balancing effect. Further analysis from the perspective of delay and packet loss rate reveals that the Zigbee protocol used by the gateway in the SCI system of this study has reached the maximum packet loss rate of $0.1 \%$ specified in the CCSA standard [32, 32]. The delay requirement is less than $10 \mathrm{~ms}$, which shows that the Zigbee protocol applied for the SCI processing in the study can meet the requirements in terms of delay and packet loss rate.

\section{Conclusion}

As the process of urbanization is accelerating, some social problems continue to emerge, such as traffic jams, inefficient urban management, and imperfect environmental monitoring systems. IoT and cloud computing are adopted in this study to 
build an SCI system by designing the applications in smart environment monitoring, smart transportation, and smart epidemic prevention of the smart cities [34, 35]. Analysis on performance of cloud computing and IoT technologies in the SCI system reveals that the VM deployment algorithm optimized by the multi-objective combination of this study can greatly reduce the number of physical servers in the SCI system and enable the server cluster to achieve better load balancing effects. In addition, the Zigbee protocol used by the IoT gateway has reached higher requirements in latency and PLR, providing experimental references for the later construction of the SCI system [36].

However, there are some limitations for this study. For example, the perception layer of the urban IoT solves the data acquisition in the human world and the physical world. The ubiquitous network and signal collection and analysis technologies are constantly improving, which continuously enriches the perception ability of the city. Therefore, in the follow-up research, it will further provide more practical solutions for the construction of related information systems and specific applications in various fields in smart city based on the development and changes of science and technology, making the system more practical.

\section{References}

1. Jararweh Y, Otoum S, AlRidhawi I (2020) Trustworthy and sustainable smart city services at the edge[J]. Sustain Cities Soc 62:102394

2. Rahman MA, Rashid MM, Hossain MS et al (2019) Blockchain and IoT-based cognitive edge framework for sharing economy services in a smart city[J]. IEEE Access 7:18611-18621

3. Bestak R, Smys S (2019) Big data analytics for smart cloud-fog based applications[J]. J Trends Comput Sci Smart Technol (TCSST) 1(02):74-83

4. Jeong YS, Park JH (2019) IoT and smart city technology: Challenges, opportunities, and solutions[J]. JIPS 15(2):233-238

5. Teng H, Dong M, Liu Y et al (2021) A low-cost physical location discovery scheme for large-scale internet of things in smart city through joint use of vehicles and UAVs[J]. Future Gener Comput Syst 118:310-326

6. Wang D, Zhong D, Souri A (2021) Energy management solutions in the internet of things applications: Technical analysis and new research directions[J]. Cogn Syst Res 67:33-49

7. Fu H, Wang M, Li P et al (2019) Tracing knowledge development trajectories of the internet of things domain: A main path analysis[J]. IEEE Trans Ind Inform 15(12):6531-6540

8. Miladinovic I, Schefer-Wenzl S (2018) Dynamic allocation of smart city applications[J]. Open J Internet Things (OJIOT) 4(1):144-149

9. Yassine A, Singh S, Hossain MS et al (2019) IoT big data analytics for smart homes with fog and cloud computing[J]. Future Gener Comput Syst 91:563-573

10. Bresciani S, Ferraris A, Del Giudice M (2018) The management of organizational ambidexterity through alliances in a new context of analysis: Internet of Things (IoT) smart city projects[J]. Technol Forecast Soc Chang 136:331-338

11. Qian Y, Wu D, Bao W et al (2019) The internet of things for smart cities: Technologies and applications[J]. IEEE Network 33(2):4-5

12. Chen Y, Hu S, Mao H et al (2020) Application of the best evacuation model of deep learning in the design of public structures[J]. Image Vis Comput 102:103975

13. Watson A, Musova Z, Machova V et al (2020) Internet of things-enabled smart cities: big datadriven decision-making processes in the knowledge-based urban economy[J]. Geopolit Hist Int Relat 12(1):94-100

14. Lv Z, Li X, Wang W et al (2018) Government affairs service platform for smart city[J]. Future Gener Comput Syst 81:443-451 
15. Hossain SKA, Rahman MA, Hossain MA (2018) Edge computing framework for enabling situation awareness in IoT based smart city[J]. J Parallel Distrib Comput 122:226-237

16. Giannakoulias A (2019) Cloud computing security: protecting cloud-based smart city applications[J]. J Smart Cities 2(1):41-52

17. Javadzadeh G, Rahmani AM (2020) Fog computing applications in smart cities: a systematic survey[J]. Wirel Netw 26(2):1433-1457

18. Hakak S, Khan WZ, Gilkar GA et al (2020) Securing smart cities through blockchain technology: architecture, requirements, and challenges [J]. IEEE Netw 34(1):8-14

19. Xu X, Huang Q, Yin X et al (2020) Intelligent offloading for collaborative smart city services in edge computing [J]. IEEE Internet Things J 7(9):7919-7927

20. Lv Z, Chen D, Lou R et al (2021) Intelligent edge computing based on machine learning for smart city [J]. Futur Gener Comput Syst 115:90-99

21. Darwish A, Hassanien AE, Elhoseny M et al (2019) The impact of the hybrid platform of internet of things and cloud computing on healthcare systems: opportunities, challenges, and open problems [J]. J Ambient Intell Humaniz Comput 10(10):4151-4166

22. Stergiou C, Psannis KE, Kim BG et al (2018) Secure integration of IoT and cloud computing[J]. Future Gener Comput Syst 78:964-975

23. Wu SM, Chen T, Wu YJ et al (2018) Smart cities in Taiwan: a perspective on big data applications[J]. Sustainability 10(1):106

24. Esposito C, Ficco M, Gupta BB (2021) Blockchain-based authentication and authorization for smart city applications[J]. Inf Process Manag 58(2):102468

25. AlZubi AA, Alarifi A, Al-Maitah M et al (2021) Multi-sensor information fusion for internet of things assisted automated guided vehicles in smart city[J]. Sustain Cities Soc 64:102539

26. Jiang D (2020) The construction of smart city information system based on the internet of things and cloud computing[J]. Comput Commun 150:158-166

27. Liu D, Zhen H, Kong D et al (2021) Sensors anomaly detection of industrial internet of things based on isolated forest algorithm and data compression. Sci Program 2021:234-243

28. Tang S, Shelden DR, Eastman CM et al (2019) A review of building information modeling (BIM) and the internet of things (IoT) devices integration: present status and future trends[J]. Autom Constr 101:127-139

29. Al-Smadi AM, Alsmadi MK, Baareh AK et al (2019) Emergent situations for smart cities: a survey. Int J Electr Comput Eng (2088-8708) 9:103-120

30. Bhatti F, Shah MA, Maple C et al (2019) A novel internet of things-enabled accident detection and reporting system for smart city environments[J]. Sensors 19(9):2071

31. Shafik W, Matinkhah SM, Ghasemzadeh M (2020) Internet of things-based energy management, challenges, and solutions in smart cities[J]. J Commun Technol Electron Comput Sci 27:1-11

32. Anuradha M, Jayasankar T, Prakash NB et al (2021) IoT enabled cancer prediction system to enhance the authentication and security using cloud computing $[\mathrm{J}]$. Microprocess Microsyst 80:103301

33. Chen J, Ye X, Chen M, Liang Y (2019) Bibliometric analysis of the papers on urban education. Libr Hi Tech 37(4):894-905

34. Chen M, Liu Q, Huang S, Dang C (2020) Environmental cost control system of manufacturing enterprises using artificial intelligence based on value chain of circular economy. Enterp Inf Syst. https://doi.org/10.1080/17517575.2020.1856422

35. Shen C-W, Min C, Wang C-C (2019) Analyzing the trend of O2O commerce by bilingual text mining on social media. Comput Hum Behav 101:474-483. https://doi.org/10.1016/j.chb.2018.09.031

36. Wen X (2020) Using deep learning approach and IoT architecture to build the intelligent music recommendation system. Soft Comput. https://doi.org/10.1007/s00500-020-05364-y

Publisher's Note Springer Nature remains neutral with regard to jurisdictional claims in published maps and institutional affiliations. 